\title{
NEURONE: oNlinE inqUiRy experimentatiON systEm
}

\author{
Roberto González-Ibáñez ${ }^{1} \quad$ Daniel Gacitúa $^{1} \quad$ Eero Sormunen $^{2} \quad$ Carita Kiili $^{3}$ \\ ${ }^{1}$ Departamento de Ingeniería Informática, Universidad de Santiago de Chile, Santiago, Chile \\ ${ }^{2}$ Faculty of Communication Sciences, University of Tampere, Tampere, Finland \\ ${ }^{3}$ Department of Education, University of Oslo, Oslo, Norway \\ 1\{roberto.gonzalez.i, daniel.gacitua\}@usach.cl, 2eero.sormunen@uta.fi, ${ }^{3}$ C.p.s.kiili@iped.uio.no
}

\begin{abstract}
In this poster we introduce NEURONE, an open source Webbased system for assessing online inquiry competences (OIC). NEURONE is a fully customizable solution that can be adapted to different research conditions through eight modules and a set of tools. To date, NEURONE has been successfully used to implement, deploy, and run an online assessment contextualized in a pretest-posttest study design, in which nearly 1400 sessions were conducted with 350 elementary school students in their early adolescence.
\end{abstract}

\section{Keywords}

Online inquiry competences, information literacy, assessment, tests, elementary schools.

\section{INTRODUCTION}

Information literacy (IL), the competences of searching, evaluating and using information sources, is a traditional research theme in library and information science. In today's Web dominated information environment, the assessment of online inquiry competences (OIC), as part of IL, is an open and hot research problem.

Library associations have developed standards for IL competences (e.g., American Library Association, 2000). Competency standards explicate the goals for IL instruction and the criteria for the assessment of learning outcomes. In this sense, a key challenge is how to design and implement valid and reliable tests that can be applied effectively to assess OIC.

To address the above challenge, we have created NEURONE (oNlinE inqUiRy experimentatiON systEm), an open source system to implement and conduct tests focused on assessing OIC. The following sections of this poster focuses on describing background aspects, NEURONE architecture, a brief discussion of its success, and some considerations for the actual visual presentation session.

\section{BACKGROUND}

Usually online research takes place when readers engage in complex processes of inquiry and problem solving. We refer to this broader task as online inquiry. In online inquiry, a person seeks answers to questions and uses the Internet to

ASIST 2017, Washington, DC | Oct. 27-Nov 1, 2017

Authors retain Copyright. learn from sources retrieved (Leu et. al., 2015). Interestingly, studies on exploratory search and searching as learning (Rieh et al., 2014) focus on similar phenomena.

Different approaches have been devised to test IL. In particular, fixed-choice tests, performance assessments, and rubrics, are well-documented alternatives to assess OIC (Oakleaf, 2008). Each of these approaches supposes pros and cons. For instance, fixed-choice tests are efficient to use, but they fail in measuring higher-level thinking skills. Conversely, performance-based assessments may be good at capturing higher-level thinking skills, but they tend to be expensive to implement.

Some attempts have been made to create technology to aid the assessment of OIC. For instance, the international test PISA includes a component to capture data about students' capacity to select and evaluate information (OECD, 2015). Others have built tools such as Meta-Analyzer to serve specific research needs (Tseng et al., 2009). These kinds of systems provide tools to assess both the end products and the process of online inquiry.

The approach introduced in this poster is based on this kind of systems, however, it expands the views to OICs and it is presented as an open source solution for researchers and educators.

\section{NEURONE}

Contextualized in an international research project, which focuses on building a deeper understanding of students' OIC and developing an instructional model to enhance OIC learning in primary education (iFuCo project), we designed NEURONE, a Web-based solution to implement OIC performance assessments (www.neurone.info).

NEURONE is an all-in-one distributed solution to implement and conduct OIC tests. The system comprises eight components (modules and set of tools), namely, (1) a search engine that operates on a user-defined corpus of webpages, and which appearance mimics that of well-known search engines; (2) tools for information gathering, which enable subjects to bookmark web pages and collect snippets from them for later usage; (3) a customizable form to let subjects express their critical evaluation of information sources; (4) synthesis tools in which subjects can revisit 
information gathered and write a synthesis relying on multiple sources; (5) a powerful logger of users' search behaviors, which enable researchers and assessors to take a closer look to online inquiry as a process; (6) a module to automatically control session flow according to a test protocol; (7) a module to build customized forms that can be used to create surveys contextualized within the test; and (8) a general user interface that operates as a common container of all modules and tools. NEURONE's conceptual architecture is depicted in Figure 1.

Besides the above components, NEURONE has several features that make it a powerful system. First of all, it follows internationalization standards (i18n), as a result, its user interface labels can be easily translated into different languages. Second, despite the kind of functionalities supported by NEURONE, the system does not require browser extensions to work. For example, the logger component can automatically collect data such as keystrokes, mouse actions (i.e., clicks, scrolls and movements), queries, pages visited, time spent in pages, and click through behavior, to name a few. However, to operate properly, it requires a browser that supports ECMAScript 6 . Third, at the communication level, NEURONE supports both WebSockets and AJAX, thus it is flexible depending on the network conditions where tests will be conducted. Fourth, the flow administration component let researchers to express a test as a set of stages (e.g., search, information gathering, critical evaluation) in a particular order and under specific constraints (e.g. time, relevant documents required, etc.).

NEURONE was implemented with state-of-the-art technology for Web development, namely, Node.js, Meteor, MongoDB, Apache Solr, AngularJS, and Docker, to name a few. With the hope that others can use and extend NEURONE, we release it as an open source solution. Therefore, researchers can use it as is or they can change the source code to meet their particular needs. To ease both processes, fully detailed documentation (in English) is provided for both researchers and system administrators.

\section{SUCCESS CASE}

NEURONE has been successfully used in the context of the iFuCo project. To date, nearly 1400 sessions have been conducted in Finland with approximately 350 elementary school students (between 11 and 12 years old) performing four tasks in the context of a pretest-posttest study design. As a result, approximately 11 million records were collected (surveys, search logs, synthesis responses, critical evaluation, etc.).

Preliminary results of OIC assessments and the design of an evaluation framework have been published elsewhere (Sormunen et al., 2017). Moreover, data is now being used to build prediction and classification models of students' search performance using behavioral data.

\section{NEXT STEPS}

NEURONE and its documentation will be available to download through a dedicated Website and an online Git

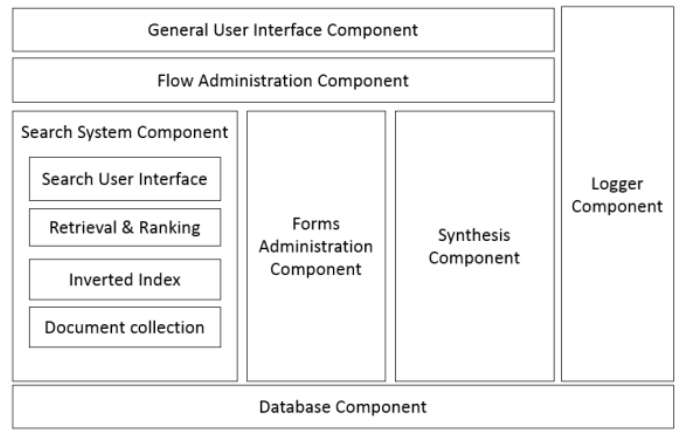

Figure 1. NEURONE's conceptual architecture.

repository. Moreover, additional user interface components are being developed to ease the process of setting up a new study (e.g., loading and indexing of a corpus of documents, changing tasks descriptions, changing language, customizing session flow). Furthermore, with the aim of replicating our success case in a different cultural context, NEURONE will be used during the second half of 2017 in a study in Chile with nearly 500 elementary school students.

\section{FORMAT OF THE PRESENTATION}

During the Visual Presentation at ASIS\&T 2017, a live demonstration of NEURONE will be performed. Audience will be able to use an instance of NEURONE and take a look to the kind of data captured by NEURONE. Moreover, examples of data analysis and preliminary results of the success case will be discussed with the audience.

\section{ACKNOWLEDGMENTS}

The study was part of the iFuCo project funded by the Academy of Finland (grant's no. 294186) and the National Commission for Scientific and Technological Research (CONICYT) in Chile (grant's AKA/EDU-03).

\section{REFERENCES}

American Library Association. (2000). Information Literacy Competency Standards for Higher Education. Chicago, IL.

Leu, D. J., Forzani, E., Rhoads, C., Maykel, C., Kennedy, C., \& Timbrell, N. (2015). The new literacies of online research and comprehension: Rethinking the reading achievement gap. Reading Research Quarterly, 50(1), 37-59.Oakleaf, 2008.

Oakleaf, M. (2009). Using rubrics to assess information literacy: An examination of methodology and interrater reliability. Journal of the American Society for Information Science and Technology, 60(5), 969983.

OECD (2015). Using log-file data to understand what drives performance in PISA (Case Study), PISA, OECD Publishing, Paris, DOI: 10.1787/9789264239555-10-en

Rieh, S. Y., Gwizdka, J., Freund, L., \& Collins- Thompson, K. (2014). Searching as learning: Novel measures for information interaction research. Proceedings of the American Society for Information Science and Technology, 51(1), 1-4.

Sormunen, E., González-Ibáñez, R., Kiili, C., Leppänen, P.H.T., MikkiläErdmann, M., Erdmann, N. \& Escobar-Macaya, M. (2017). A Performance-based Test for Assessing Students' Online Inquiry Competences in Schools. Submitted to the European Conference on Information Literacy (ECIL), 18-21 September 2017, Saint-Malo, France. 10 pages

Tseng, J. C., Hwang, G. J., Tsai, P. S., \& Tsai, C. C. (2009). Meta-analyzer: A web-based learning environment for analyzing student information searching behaviors. International Journal of Innovative Computing, Information and Control, 5(3), 567-579. 